$$
\begin{aligned}
& F_{89}\left(x_{8}\right)=f\left(x_{8}\right) \leqq F_{k_{0}}\left(x_{8}\right), \\
& F_{89}\left(x_{9}\right)=f\left(x_{9}\right)<F_{k_{0}}\left(x_{9}\right),
\end{aligned}
$$

so that, by Theorem 1 and its corollary,

$$
F_{89}(x)<F_{k_{0}}(x), \quad\left(x_{8}<x<b\right) ;
$$

in particular,

$$
F_{89}\left(x_{7}\right)<F_{k_{0}}\left(x_{7}\right) .
$$

Now (41) contradicts (39) and (40).

The Rice Institute

\title{
SUFFICIENT CONDITIONS FOR A NON-REGULAR PROBLEM IN THE CALCULUS OF VARIATIONS*
}

\section{G. M. EWING}

1. Introduction. Given $J=\int_{x_{1}}^{x_{2}} f\left(x, y, y^{\prime}\right) d x$, it is well known that a minimizing curve satisfies the necessary conditions of Euler, Weierstrass, and Legendre, which we shall designate as I, II, and III, $\dagger$ respectively. If further, $f_{y^{\prime} y^{\prime}}\left(x, y, y^{\prime}\right) \neq 0$ on the minimizing curve, the Jacobi condition IV is necessary, while the stronger set of conditions $\mathrm{I}, \mathrm{II}_{b}^{\prime}, \mathrm{III}^{\prime}$, and $\mathrm{IV}^{\prime} \ddagger$ are sufficient for a strong relative minimum.

The purpose of this study is to obtain a set of sufficient conditions for a curve without corners along which $f_{y^{\prime} y^{\prime}}$ may have zeros. Since the classical theory gives only the necessary conditions I, II, and III, we wish to obtain a Jacobi condition; and with this in view, introduce the integral

$$
\begin{array}{r}
L \equiv \int_{x_{1}}^{x_{2}} \phi\left(x, y, y^{\prime}\right) d x, \phi\left(x, y, y^{\prime}\right) \equiv f\left(x, y, y^{\prime}\right)+k^{2}\left[y^{\prime}-e^{\prime}(x)\right]^{2}, \\
\left(x_{1} \leqq x \leqq x_{2}, k \leqq 0\right)
\end{array}
$$

by means of which we find a necessary condition that we shall call $I V_{L}^{\prime}$. Suitably strengthened, this becomes $I_{L b}^{\prime}$ and the set of conditions $\mathrm{I}, \mathrm{II}_{b}, \mathrm{III}_{b}$, and $\mathrm{IV}_{L b}^{\prime}$ are found sufficient for an improper strong relative minimum.

* Presented to the Society, November 27, 1936.

$\dagger$ G. A. Bliss, Calculus of Variations, 1925, pp. 130-132.

$\ddagger$ Bliss, loc. cit., pp. 134-135. 
It appears likely that analogous results can be obtained for other problems in the Calculus of Variations and I hope to discuss some of these at a later time.

2. A Jacobi Necessary Condition. If $E: y=e(x)$ furnishes at least an improper strong relative minimum for $J$, it furnishes a proper strong relative minimum for $L$. Furthermore, if $E$ minimizes $J$ it satisfies III for $J$. This implies that it satisfies III' for $L$, since $\phi_{y^{\prime} y^{\prime}}=f_{y^{\prime} y^{\prime}}+2 k^{2}$; and the classical treatment then shows that it must satisfy IV for L.

If $E$ satisfies IV (or $I^{\prime}$ ) for every $L$, we shall say that it satisfies the condition $\mathrm{IV}_{L}$ (or $I V_{L}^{\prime}$, respectively) for $J$. Clearly $I V_{L}$ is necessary. We now show that the same is true of $I V_{L}^{\prime}$.

We write the parameter in $L$ in the form $k^{2}=\left(a^{2}+\alpha\right) / 2, a \neq 0$, $\alpha>-a^{2}$, and consider the Jacobi differential equations*

$$
\begin{aligned}
q u^{\prime \prime}+r u^{\prime}+s u & =0 \\
\left(q+a^{2}+\alpha\right) u^{\prime \prime}+r u^{\prime}+s u & =0
\end{aligned}
$$

for $J$ and $L$, where $q=f_{y^{\prime} y^{\prime}}\left[x, e(x), e^{\prime}(x)\right] \geqq 0$ in the closed interval $\left[x_{1}, x_{2}\right]$ from III, and $r$ and $s$ are other known functions of $x$. Since $q$ may vanish in $\left[x_{1}, x_{2}\right]$, the usual existence theorems can not be applied to (1). They do apply to (2), however, the general solution of which for $\alpha=0$ is $u=c_{1} u_{1}(x)+c_{2} u_{2}(x)$, where the $u^{\prime}$ s constitute a fundamental system and are of class $C^{\prime \prime} \dagger$ in $\left[x_{1}, x_{2}\right]$. $\Delta\left(x, x_{1}\right)= \pm u_{2}\left(x_{1}\right) u_{1}(x) \mp u_{1}\left(x_{1}\right) u_{2}(x)$ is a particular solution vanishing at $x=x_{1}$. By hypothesis, $E: \quad y=e(x)$ is a minimizing curve satisfying $I_{L}$ so that, by proper choice of signs, $\Delta\left(x, x_{1}\right)$ is positive in the interval $x_{1}<x<x_{2}$.

For every admissible $\alpha$ (that is, $\alpha>-a^{2}$ ) there exists a solution $\Delta\left(x, x_{1}, \alpha\right)$ of (2) vanishing at $x=x_{1}$ and such that $\Delta^{\prime}\left(x_{1}, x_{1}, \alpha\right)$ $=\Delta^{\prime}\left(x_{1}, x_{1}\right)$, where $\Delta^{\prime \prime}\left(x, x_{1}, \alpha\right)$ is continuous in $x$ and of class $C^{\prime}$ in $\alpha . \ddagger$

We next study the related equation

$$
\left(q+a^{2}\right) u^{\prime \prime}+r u^{\prime}+s u=-\alpha \Delta^{\prime \prime}\left(x, x_{1}, \alpha\right)
$$

* Oskar Bolza, Vorlesungen uber Variationsrechnung, 1933, p. 60.

$\uparrow$ That is, they have continuous second derivatives. Bolza, loc. cit., p. 14.

$\ddagger$ Replace (2) by the system $u^{\prime}=v$ and $\left(q+a^{2}+\alpha\right) v^{\prime}+r v+s u=0$, and apply the existence theorem given by Bolza, loc. cit., p. 187. 
whose general solution can, by the method of variation of parameters, be expressed in the form

$$
u=c_{1} u_{1}(x)+c_{2} u_{2}(x)+\alpha A(x, \alpha),
$$

where

$$
\begin{gathered}
A(x, \alpha)=u_{1}(x) \int_{x_{1}}^{x} \frac{\Delta^{\prime \prime}\left(x, x_{1}, \alpha\right) u_{2}(x) d x}{\left(q+a^{2}\right) D(x)} \\
-u_{2}(x) \int_{x_{1}}^{x} \frac{\Delta^{\prime \prime}\left(x, x_{1}, \alpha\right) u_{1}(x) d x}{\left(q+a^{2}\right) D(x)} \\
D(x) \equiv\left|\begin{array}{cc}
u_{1}(x) & u_{2}(x) \\
u_{1}^{\prime}(x) & u_{2}^{\prime}(x)
\end{array}\right| \neq 0 \text { in the closed interval }\left[x_{1}, x_{2}\right]{ }^{*}
\end{gathered}
$$

$\Delta\left(x, x_{1}, \alpha\right)$, as a particular solution of (3), can be represented in the form (4); and, since it vanishes for $x=x_{1}$, we obtain

$$
\Delta\left(x, x_{1}, \alpha\right)=\lambda \Delta\left(x, x_{1}\right)+\alpha A(x, \alpha),
$$

where in general $\lambda$ is a function of $\alpha$. Clearly $\lambda(0)=1$.

$E$ satisfies $I_{L}$ by hypothesis. If it fails to satisfy IV' for the $L$ corresponding to $\alpha=0$, we have

$$
\Delta\left(x_{2}, x_{1}, 0\right)=\lambda(0) \Delta\left(x_{2}, x_{1}\right)=\Delta\left(x_{2}, x_{1}\right)=0,
$$

while, if a second $\alpha \neq 0$ has the same property, we have

$$
\Delta\left(x_{2}, x_{1}, \alpha\right)=\lambda(\alpha) \Delta\left(x_{2}, x_{1}\right)+\alpha A\left(x_{2}, \alpha\right)=\alpha A\left(x_{2}, \alpha\right)=0 .
$$

This requires

$$
A\left(x_{2}, \alpha\right)=0
$$

But

$$
\begin{aligned}
& A\left(x_{2}, \alpha\right)=\int_{x_{2}}^{x_{1}} \frac{\Delta^{\prime \prime}\left(x, x_{1}, \alpha\right) \Delta\left(x, x_{2}\right)}{\left(q+a^{2}\right) D(x)} d x \\
&=\frac{\Delta\left(\bar{x}, x_{2}\right)}{\left(\bar{q}+a^{2}\right) D(\bar{x})} \int_{x_{2}}^{x_{1}} \Delta^{\prime \prime}\left(x, x_{1}, \alpha\right) d x \\
&=\frac{\Delta\left(\bar{x}, x_{2}\right)\left[\Delta^{\prime}\left(x_{1}, x_{1}, \alpha\right)-\Delta^{\prime}\left(x_{2}, x_{1}, \alpha\right)\right]}{\left(\bar{q}+a^{2}\right) D(\bar{x})}, \\
&\left(x_{1}<\bar{x}<x_{2} ; \bar{q}=q(\bar{x})\right),
\end{aligned}
$$

* Bolza, loc. cit., p. 75. 
where $\Delta\left(x, x_{2}\right)$ is written for $u_{2}\left(x_{2}\right) u_{1}(x)-u_{1}\left(x_{2}\right) u_{2}(x)$. This fraction can not vanish, the first factor in the numerator being different from zero by $\mathrm{IV}_{L}$, the second factor being the difference between two terms of opposite sign. Thus (6) is false; and there is at most one $L$, namely the one for which $\alpha=0$, for which $E$ fails to satisfy IV'.

If $\Delta\left(x_{2}, x_{1}, 0\right)=0$, we have $\Delta\left(x_{2}, x_{1}, \alpha\right)=\alpha A\left(x_{2}, \alpha\right)$ from (5). Furthermore $\Delta\left(x_{2}, x_{1}, \alpha\right)$ must then have a minimum of zero for $\alpha=0$; so that its derivative, which is $A\left(x_{2}, 0\right)$, must vanish. This is a special case of (6), which has been proved to be false, so that $I V_{L}^{\prime}$ is a necessary condition.

3. Sufficient Conditions for a Minimum for $L$. We assume an arc $E: \quad y=e(x)$ satisfying the necessary conditions I, II, III, and $I V_{L}^{\prime}$ for $J$. If $I I$ is strengthened to $\mathrm{II}_{b}$, we can show that this arc satisfies the classical sufficient conditions for $L$.

Comparing the Euler equations, we see that if $E$ satisfies I for $J$ it does the same for $L$. The $E$-functions $\dagger$ for the two problems are related by the equation

$$
E_{L}\left(x, y, y^{\prime}, Y^{\prime}\right) \equiv E_{J}\left(x, y, y^{\prime}, Y^{\prime}\right)+k^{2}\left(y^{\prime}-Y^{\prime}\right)^{2}
$$

so that $\mathrm{II}_{b}$ for $J$ implies $\mathrm{II}_{b}^{\prime}$ for $L$. We have seen in $\$ 2$ that III for $J$ implies III' for $L$ and the condition IV $_{L}^{\prime}$ requires $I V^{\prime}$ for $L$ as a matter of definition.

Hence $E$ furnishes a proper strong minimum to $L$ relative to a certain $(x, y)$ region $R$, which in general depends on $k . \ddagger$

4. Sufficient Conditions for an Improper Strong Relative Minimum for $J$. We must find how to strengthen our conditions so as to insure a field $\$$ which is independent of $k$. To that end we replace III by III $_{b}$ and consider the line $\Lambda: x=x_{1}, y=n \lambda-y_{1}$, together with a slope function $P(\lambda) \equiv m \lambda+e^{\prime}\left(x_{1}\right)$. The extremals for $L$ are $y=y(x, a, b, \alpha)$, and the equations

* $\Delta\left(x_{2}, x_{1}, \alpha\right)>0$ for $\alpha \neq 0$ by IV $\mathrm{IV}_{L}$ and the choice of signs preceding equation

$\dagger$ This is the only direct reference to the $E$-function. There need be no confusion with our notation for the curve $E: y=e(x)$.

$\ddagger$ If $E$ satisfies III' and IV. but not IV $^{\prime}$, for $J, R$ reduces to the curve $E$ as $k$ approaches zero.

$\S$ Bliss, loc. cit., pp. 132-33. 


$$
\begin{aligned}
n \lambda+y_{1}-y\left(x_{1}, a, b, \alpha\right) & =0, \\
m \lambda+e^{\prime}\left(x_{1}\right)-y^{\prime}\left(x_{1}, a, b, \alpha\right) & =0,
\end{aligned}
$$

define $a=a(\lambda, \alpha)=\bar{a}(y, \alpha)$, and $b=b(\lambda, \alpha)=\bar{b}(y, \alpha) *$ for any admissible $\alpha$ and for every $y$ for which $\left(x_{1}, y\right)$ is in the region where III $_{b}$ holds. These implicit functions are of at least class $C^{\prime}$ in their respective variables. We thus have a family of extremals of parameter $\lambda$ for each admissible $\alpha$,

$$
y=\phi(x, \lambda, \alpha) \equiv y[x, a(\lambda, \alpha), b(\lambda, \alpha), \alpha],
$$

intersecting $\Lambda$ and including $E$ for $\lambda=0$. We wish this family to furnish a field.

If there exists an $x,\left(x_{1}<x \leqq x_{2}\right)$, such that $\phi\left(x, \lambda_{1}, \alpha\right)$ $-\phi\left(x, \lambda_{2}, \alpha\right)=0$, there is a $\bar{\lambda},\left(\lambda_{1}<\bar{\lambda}<\lambda_{2}\right)$, such that

$$
\phi_{\lambda}(x, \bar{\lambda}, \alpha)=y_{a} \frac{\partial a}{\partial \lambda}+\left.y_{b} \frac{\partial b}{\partial \lambda}\right|_{\lambda=\bar{\lambda}}=0 .
$$

This can be expressed in the form $\dagger$

$$
\begin{gathered}
\frac{n}{D_{1}}\left|\begin{array}{cc}
y_{a}(x) & y_{b}(x) \\
y_{a}^{\prime}\left(x_{1}\right) & y_{b}^{\prime}\left(x_{1}\right)
\end{array}\right|-\frac{m}{D_{1}}\left|\begin{array}{ll}
y_{a}(x) & y_{b}(x) \\
y_{a}\left(x_{1}\right) & y_{b}\left(x_{1}\right)
\end{array}\right|, \\
D_{1}=\left|\begin{array}{cc}
y_{a}\left(x_{1}\right) & y_{b}\left(x_{1}\right) \\
y_{a}^{\prime}\left(x_{1}\right) & y_{b}^{\prime}\left(x_{1}\right)
\end{array}\right| \neq 0 . \ddagger
\end{gathered}
$$

We shall say that $E$ satisfies the condition $I_{L b}^{\prime}$ if constants $\delta>0, \eta>0$, and $A$ exist such that $\S$

$$
\Delta\left(x, x_{1}, y, \alpha\right) \equiv\left|\begin{array}{ll}
\bar{y}_{a}(x) & \bar{y}_{b}(x) \\
\bar{y}_{a}\left(x_{1}\right) & \bar{y}_{b}\left(x_{1}\right)
\end{array}\right|
$$

is, in absolute value, greater than $\delta$ in the region $x_{1}<x \leqq x_{2}$, $\left|y-y_{1}\right| \leqq \eta, A \geqq \alpha>-a^{2}$. The first determinant in (7) has a finite limit as $n$ approaches zero; and hence, if $n$ is small in absolute value, $I V_{L b}^{\prime}$ insures that the expression will not vanish and that no two extremals of the family pass through the same

* $a, b, \bar{a}$, and $\bar{b}$ also depend on $m$ and $n$, which are omitted in the notation. $\dagger y_{a}(x), \cdots$ are written for $y_{a}[x, a(\lambda, \alpha), b(\lambda, \alpha), \alpha], \cdots$.

$\ddagger$ The method used by Bolza, loc. cit., pp. 73-75, shows that $D_{1} \neq 0$.

$\S \bar{y}_{a}(x), \cdots$ are written for $y_{a}[x, \bar{a}(y, \alpha), \bar{b}(y, \alpha) \alpha], \cdots$. 
point. This condition also requires $\phi$ to be strictly monotone in $\lambda$ for a given $x$ and $\alpha$, so that an extremal of the family passes through each point of a certain region $F$ about $E$. The region $F$ is a field and is independent of $k$ (that is, of $\alpha$ ).*

Finally, if $E$ satisfies $\mathrm{I}, \mathrm{II}_{b}, \mathrm{III}_{b}$, and $\mathrm{IV}_{L b}^{\prime}$, we have $L(E)$ $<L(C)$ for every $C \neq E$ in $F$. But

$$
L(C)=J(C)+\epsilon, \quad \epsilon>0, \quad \lim _{k \doteq 0} \epsilon=0 .
$$

Furthermore $L(E)=J(E)$, so that $J(E)<J(C)+\epsilon$, and finally $J(E) \leqq J(C)$.

5. Applications. The line $y=0$ is an extremal for a problem involving any one of the following integrands:

$$
\begin{aligned}
f & \equiv M(x, y)+N(x, y) y^{\prime}, \quad M_{y}=N_{x}, \\
f & \equiv x^{2}+y^{2}+y y^{\prime}, \dagger \\
f & \equiv y^{\prime 4} .
\end{aligned}
$$

Our sufficient conditions for an improper minimum are met by $y=0$ in each case, but III' is not met for any of them.

The University of Missouri

* Condition $\mathrm{IV}_{L b}^{\prime}$ could be replaced by the following. There exist constants $\eta>0, \xi>0$, and $A$ such that $E$ satisfies III for $x_{0} \leqq x \leqq x_{2}, x_{0}=x_{1}-\xi$ and such that $\Delta\left(x, x_{0}, y, \alpha\right) \neq 0$ for $x_{0}=x_{1}-\xi, x_{0}<x \leqq x_{2},\left|y-y_{1}\right| \leqq \eta, A \geqq \alpha>-a^{2}$. See Bolza, loc. cit., bottom p. 103.

$\dagger$ An example given by Bolza, loc. cit., p. 35 . 